\title{
Estrategias para mitigar impactos negativos del trabajo infantil
}

\author{
María Fernanda Serrano-Guzmán* \\ Mónica Natalia Serrano-Guzmán** \\ Marisol Mesa-Rivera ${ }^{* *}$ \\ Marina del Mar Mármol-Ríos****
}

\footnotetext{
Doctora en Ingeniería Civil, Universidad de Puerto Rico. Profesora titular, Facultad de Ingeniería civil, Pontificia Universidad Javeriana, Cali, Colombia.

Correo electrónico:

maria.serrano@javerianacali.edu.co

** Magíster en Educación. Directora, Colegio Juan XXIII, Macaravita, Colombia. Correo electrónico:

doctora-serrano@hotmail.com

*** Magíster en Educación, Universidad Pontificia Bolivariana. Secretaria de Educación para la Cultura de Antioquia, Medellín, Colombia.

Correo electrónico:

mesariveramarisol@yahoo.es

${ }^{* * *}$ Magíster en Educación. Gerencia

Departamental Colegiada de Santander,

Grupo de Vigilancia Fiscal, Bucaramanga, Colombia.

Correo electrónico:

marina.marmol@contraloria.gov.co
}

Recibido: 29 de agosto del 2014

Aprobado: 18 de diciembre del 2014

Cómo citar este artículo: María Fernanda Serrano-Guzmán, Mónica Natalia SerranoGuzmán, Marisol Mesa-Rivera y Marina del Mar Mármol-Ríos. Estrategias para mitigar impactos negativos del trabajo infantil. DIx 21. Junio de 2015. Pág. 81. doi: http://dx.doi.org/10.16925/ di.v17i21.981

\section{Resumen}

La situación económica en ciertas regiones de Colombia está propiciando el trabajo infantil como apoyo a la cadena productiva familiar. La mayoría de estudios realizados presentan las estadísticas de lo encontrado a nivel urbano, de manera que lo rural queda por fuera de los indicadores a ese respecto. Sin embargo, un estudio realizado en una comunidad rural en Santander (Colombia) reveló que el trabajo infantil que desempeñan los infanto-jóvenes está incidiendo en el desempeño escolar y en la deserción de los procesos de formación para toda la vida. Partiendo de los resultados de este estudio, se diseñaron las estrategias para minimizar las consecuencias del trabajo infantil. Como resultado, se presentan las iniciativas que pueden ofrecerse a la comunidad académica y a la sociedad, a fin de mitigar los impactos negativos ocasionados por el trabajo infantil. De igual forma, se plantea cómo es necesario para la academia hacer ajustes al currículo con la incorporación de nuevas estrategias pedagógicas, fundamentadas en el aprendizaje basado en problemas, el trabajo colaborativo y la creación de programas abiertos y a distancia. Por su parte, para la sociedad se proponen los talleres de sensibilización.

Palabras clave: aprendizaje, educación, estrategia pedagógica, trabajo infantil. 


\title{
Strategies for Mitigating Negative Impacts of Child Labor
}

\begin{abstract}
The economic situation in certain regions of Colombia encourages child labor to support the family productive chain. Most studies that have been made present the statistics found at the urban level, so that the rural situation is left out of indicators in that respect. However, one particular study undertaken in a rural community in Santander (Colombia) showed that child labor performed by children and young people is having an effect on school performance and dropout rates throughout life. Based on the results of this study, strategies were designed to minimize the effects of child labor. As a result, initiatives are presented that could be made available to the academic community and society in order to mitigate negative impacts stemming from child labor. Additionally, the need for academia to make adjustments in curricula through the incorporation of new pedagogical strategies using learning based on problems, collaborative work and the creation of open programs and learning at a distance is proposed. Sensitization workshops are also proposed for society.
\end{abstract}

Keywords: learning, education, pedagogical strategy, child labor.

\section{Estratégias para mitigar impactos negativos do trabalho infantil}

\section{Resumo}

A situação econômica em certas regiões da Colômbia está propiciando o trabalho infantil como apoio à cadeia produtiva familiar. A maioria dos estudos realizados apresenta as estatísticas do que se constata no contexto urbano e o rural fica por fora dos indicadores a esse respeito. Contudo, um estudo realizado numa comunidade rural em Santander (Colômbia) revelou que o trabalho infantil que desempenham os infantes/jovens está incidindo no desempenho escolar e no abandono dos processos de formação para toda a vida. Partindo dos resultados desse estudo, foram desenhadas as estratégias para reduzir as consequências do trabalho infantil. Além disso, evidencia-se a necessidade de a academia fazer ajustes aos currículos com a incorporação de novas estratégias pedagógicas, fundamentadas na aprendizagem baseada em problemas, no trabalho colaborativo e na criação de programas abertos e a distância. Por sua vez, para a sociedade civil, propõem-se as oficinas de sensibilização.

Palavras-chave: aprendizagem, educação, estratégia pedagógica, trabalho infantil. 


\section{INTRODUCCIÓN}

El trabajo infantil es un flagelo social del cual no escapa Colombia. De la manera como se lleva a cabo, se están vulnerando los derechos de la niñez ante la indiferencia gubernamental y, peor aún, del núcleo familiar. Sin embargo, es de resaltar cómo, si bien durante el mandato de Juan Manuel Santos se ha reconocido el carácter de esta problemática mediante la inserción de programas de erradicación del trabajo infantil, se observa que las familias aún justifican que sus hijos se vinculen a la cadena productiva familiar en edades tempranas según la idea de aceptación cultural de que es un aprendizaje al mundo laboral. Sin embargo, este trabajo no planificado de forma racional influye en la asistencia escolar y en el rendimiento de los estudiantes. ${ }^{1}$

El objetivo de este artículo es socializar las estrategias de apoyo para el joven trabajador, con el fin de mitigar los impactos negativos que ocasiona el trabajo infantil. Se espera que las políticas públicas dirigidas a la infancia y adolescencia protejan a los niños de toda explotación económica, contra toda forma de trabajo que impida su educación y su pleno desarrollo personal, familiar y social.

\section{A. Aspectos legales}

Una de las condiciones necesarias para disminuir los niveles de pobreza y desigualdad es garantizar el funcionamiento de políticas y programas de formación de capital humano. El reto del Gobierno Nacional es continuar el proceso de aprendizaje con una educación básica y media de calidad.

En este sentido, es necesario desarrollar y fortalecer el Sistema de Formación de Capital Humano, el cual consiste en:

1. Garantizar el acceso universal a la educación básica al hacer especial énfasis en la calidad del servicio, así como reducir las brechas entre prestadores públicos y privados. De igual forma, hacer hincapié en la importancia de asegurar la permanencia de los estudiantes en el sistema.

2. Mejorar la infraestructura educativa y adecuarla a los riesgos derivados del cambio climático.

1. Véase Aura Pedraza y Rocío Ribero. El trabajo infantil y juvenil en Colombia y algunas de sus consecuencias claves. Revista LAtinoamericana de Ciencias Sociales, Niñez y Juventud 1. Enero-junio de 2006. Págs. 2-28.
3. Dar especial atención al acceso en el nivel de educación media.

4. Mejorar la cobertura y pertinencia de la educación superior, y en particular de la educación técnica y tecnológica.

5. Dinamizar y mejorar la cobertura y pertinencia de la formación para el trabajo, introduciendo esquemas competitivos y de aseguramiento de la calidad. ${ }^{2}$

Estas políticas de Estado no han impedido que una gran parte de la población entre los 10 y 17 años, que se encuentra estudiando, se dedique parcialmente a su proceso de aprendizaje, lo que genera consecuencias graves para ellos, desde afectaciones a la salud hasta problemas de aprendizaje y, en el peor de los casos, la deserción escolar. ${ }^{3}$

Para sustentar el marco legal que establece los límites del trabajo infantil, se presentan algunos conceptos y legislaciones alrededor de la defensa de los derechos de niños, niñas y adolescentes que se ven inmersos en la cadena productiva familiar o que son víctimas de cualquier vulneración de sus derechos fundamentales, afectando así su proceso de aprendizaje. Dichos referidos se resumen en la tabla 1 .

Como ya se mencionó, los jóvenes adolescentes adquieren obligaciones económicas en su núcleo familiar a muy temprana edad, lo que obstruye su formación en el ámbito educativo. Colombia como Estado Social de Derecho garantiza los derechos de los niños y las niñas en el Artículo 44 de la Constitución Nacional, de la siguiente manera:

Derecho a la vida, la integridad física, la salud y la seguridad social, la alimentación equilibrada, su nombre y nacionalidad, tener una familia y no ser separados de ella, el cuidado y el amor, la educación y la cultura, la recreación y la libre expresión de su opinión.

Serán protegidos contra toda forma de abandono, violencia física o moral, secuestro, venta, abuso sexual, explotación laboral o económica y trabajos riesgosos. Gozarán también de los demás derechos consagrados

2. Cf. Departamento Nacional de Planeación. Hacia la prosperidad democrática: visión 2010-2014. BASES Del Plan NACIONAL De DesaRrollo 2010-2014. Departamento Nacional de Planeación. (2011) Pág. 253.

3. Véase Organización Internacional del Trabajo. Fortalecimiento A FAMILIAS CON NIÑOS, NIÑAS Y JÓVENES TRABAJADORES. UN CAMINO PARA LA PREVENCIÓN Y ERRADICACIÓN DEL TRABAJO INFANTIL en Colombia. (2005). Disponible en: http://ww w.ilo.org/ipec/facts/ lang--es/index.htm [De aquí en adelante oIT] 
en la Constitución, en las leyes y en los tratados internacionales ratificados por Colombia. (...)

La familia, la sociedad y el Estado tienen la obligación de velar por el cumplimiento de los derechos de la niñez. ${ }^{4}$

En dicho mandato, se establecen tres características principales respecto a los derechos de la infancia:

1. Los derechos de los niños prevalecen sobre los derechos de los demás.

2. Los derechos económicos, sociales y culturales, cuando se trata de menores de edad, tienen carácter de derechos fundamentales.

3. Existe una corresponsabilidad entre familia, sociedad y Estado en la protección de los derechos de niños y niñas, así como en el logro de su mayor nivel de desarrollo armónico e integral.

\section{B. Concepciones del trabajo infantil}

El trabajo es una condición que la mayoría de personas considera necesaria para obtener el sustento y satisfacer las necesidades básicas y algunas suntuarias del individuo. El trabajo infantil es toda actividad económica realizada por menores, por debajo de la edad mínima general de admisión al empleo especificada en cada país, cualquiera que sea su categoría ocupacional (asalariado, independiente, trabajo familiar no remunerado), y que sea física, mental, social o moralmente perjudicial o dañina para el niño, e interfiera en su escolarización. Esto es, privándole de la oportunidad de ir a la escuela, obligándole a abandonar prematuramente las aulas, o exigiendo que intente combinar la asistencia a la escuela con largas jornadas de trabajo pesado. ${ }^{5}$

Las formas de trabajo infantil son variadas, lo cual dificulta los controles que se le puedan hacer.

Tabla 1. Referentes legales

\begin{tabular}{|c|c|}
\hline Referente & Conceptos asociados \\
\hline $\begin{array}{l}\text { Constitu- } \\
\text { ción Política } \\
\text { de Colombia } \\
\text { de } 1991\end{array}$ & $\begin{array}{l}\text { Las normas internacionales de derechos humanos ratificadas por el Estado se incorporan al texto de la Constitución. } \\
\text { Desde 1991, los derechos de los niños y de las niñas están consagrados expresamente en la Constitución Nacional con } \\
\text { rango de derechos fundamentales y con carácter prevalente. } \\
\text { La vida, la integridad física, la salud y la seguridad social, la alimentación equilibrada, su nombre y nacionalidad, tener } \\
\text { una familia y no ser separados de ella, el cuidado y amor, la educación y la cultura, la recreación y la libre expresión de } \\
\text { su opinión son derechos fundamentales de los niños y las niñas. }\end{array}$ \\
\hline $\begin{array}{l}\text { Código de } \\
\text { Infancia y } \\
\text { Adolescen- } \\
\text { cia: Ley } 1098 \\
\text { de } 2006\end{array}$ & $\begin{array}{l}\text { Artículo 8. Interés superior de los niños y las niñas. Todas las personas deben garantizar la satisfacción integral y simul- } \\
\text { tánea de todos sus derechos humanos (de los niños y las niñas), que son universales, prevalentes e interdependientes. } \\
\text { Artículo 35. La edad mínima de trabajo. En Colombia para poder trabajar, los adolescentes entre los } 15 \text { y } 17 \text { años } \\
\text { requieren la respectiva autorización expedida por el inspector de trabajo o, en su defecto, por el ente territorial local, } \\
\text { y "gozarán de las protecciones laborales, consagrados en el Régimen Laboral Colombiano, las normas complementa- } \\
\text { rias, el Código de la Infancia y la Adolescencia, los tratados y convenios internacionales ratificados por Colombia y la } \\
\text { Constitución Política". } \\
\text { Artículo 40. La corresponsabilidad. Este código asegura que las empresas, comercios y demás agentes económicos } \\
\text { tienen, como miembros de la sociedad que son, la responsabilidad de "tomar parte activa en el logro de la vigencia } \\
\text { efectiva de los derechos y garantías de los niños, las niñas y los adolescentes". }\end{array}$ \\
\hline $\begin{array}{l}\text { Ley } 1453 \text { de } \\
2011\end{array}$ & $\begin{array}{l}\text { Artículo 93. Explotación de menores de edad. El que utilice, instrumentalice, comercialice o mendigue con menores } \\
\text { de edad directamente o a través de terceros incurrirá en prisión, de } 3 \text { a } 7 \text { años, y el menor será conducido al Instituto } \\
\text { Colombiano de Bienestar Familiar para aplicar las medidas de restablecimientos de derechos correspondientes. La } \\
\text { pena se aumentará a la mitad cuando el actor sea un pariente hasta el cuarto grado de consanguinidad, segundo de } \\
\text { afinidad o primero civil. }\end{array}$ \\
\hline $\begin{array}{l}\text { Plan } \\
\text { Nacional } \\
\text { Decenal de } \\
\text { Educación } \\
\text { de Colombia }\end{array}$ & $\begin{array}{l}\text { El Plan Nacional Decenal de Educación 2006-2016 (PNDE) se define como un pacto social por el derecho a la edu- } \\
\text { cación, y tiene como finalidad servir de ruta y horizonte para el desarrollo educativo del país en el próximo decenio. } \\
\text { Referente obligatorio de planeación para todos los Gobiernos e instituciones educativas, así como instrumento de } \\
\text { movilización social y política en torno a la defensa de la educación, entendida esta como un derecho fundamental de la } \\
\text { persona y como un servicio público que, en consecuencia, cumple una función social. }\end{array}$ \\
\hline
\end{tabular}

Fuente: Mónica Serrano, Marisol Mesa y Marina del Mar Mármol. IDENTIFICACión DE INTERACCIONES ACADÉMICAS Y FAMILIARES QUE EVIDENCIAN CAMBIOS EN EL DESEMPEÑO ACADÉMICO DE ADOLESCENTES QUE INTERVIENEN EN LA CADENA PRODUCTIVA FAMILIAR EN una Comunidad RURal. Tesis de Maestría. Fundación Universitaria Católica de Colombia. (2014).

4. Constitución Política de Colombia. Artículo 44. Julio 7 de 1991.

5. Véase oIT, supra, nota 3.

(Colombia). 
Según informe de la Presidencia de la República de Colombia,${ }^{6}$ el Departamento de Trabajo de Estados Unidos refirió que el trabajo infantil en Colombia se concentra en actividades de extracción minera (carbón, esmeraldas y oro); producción agrícola (café y caña de azúcar); producción industrial en las ladrilleras, y en actividades ilegales tales como la producción de coca y la pornografía infantil. Ejecutado de esta forma, el trabajo de los menores perturba el crecimiento saludable y se revierte como un determinante de los procesos de exclusión social. ${ }^{7}$ Sin embargo, no toda tarea que realicen niños y niñas puede llegar a ser considerada como trabajo infantil, ya que hay actividades que implican la colaboración de estos en el hogar u otras que aportan a su desarrollo mediante el aprendizaje desde las experiencias vividas. ${ }^{8}$

\section{Riesgos ocasionados por el trabajo infantil}

La decisión de liberar a los menores del ciclo productivo es contemporánea al carácter colonial de la economía mundial, y viene de la política de expansión europea desde el siglo xvi. ${ }^{9}$ De continuar con la modalidad del trabajo infantil actual, se obliga a repensar un nuevo concepto de niñez, enfocando preferentemente las destrezas, las motivaciones, las experiencias y los conocimientos adquiridos en lugar de centrarse en el carácter deficitario de las carencias de la infancia. ${ }^{10}$

Serrano, Mesa y Mármol ${ }^{11}$ señalan cómo el trabajo infantil repercute en el desarrollo físico, cognitivo, emocional y social del menor, y cómo de acuerdo con lo planteado por Sandoval, ${ }^{12}$ puede desencadenar

6. Cf. Presidencia de la República. Presidente Santos resalta informe del Departamento de Trabajo de EEUU sobre aVanCES DE COLOMBIA PARA ELIMINAR LAS PEORES FORMAS DE TRABAJO INFANTIL. (Octubre 1 de 2013). Disponible en: http://wsp.presidencia.gov.co/Prensa/2013/Octubre/Paginas/20131001_04-Presidente-Santos-informe-Departamento-Trabajo-EEUU-Colombia-eliminar-peores-formas-trabajo-infantil.aspx

7. Véase Silvio Feldman, Emilio García y Hege Araldsen. Los niÑos QUE TRABAJAN. Unicef Argentina. (1997).

8. Véase Mónica Serrano, Marisol Mesa y Marina del Mar Mármol. IDENTIFICACIÓN DE INTERACCIONES ACADÉMICAS Y FAMILIARES QUE EVIDENCIAN CAMBIOS EN EL DESEMPEÑO ACADÉMICO DE ADOLESCENTES QUE INTERVIENEN EN LA CADENA PRODUCTIVA FAMILIAR EN UNA COMUNIDAD RURAL. Tesis de Maestría. Fundación Universitaria Católica de Colombia. (2014).

9. Cf. Zandra Pedraza Gómez. El trabajo infantil en clave colonial: consideraciones histórico-antropológicas. Revista NómAdAs 26. Abril de 2007. Págs. 80-90.

10. Id.

11. Véase Serrano, Mesa y Mármol, supra, nota 8.

12. Véase Antonio Sandoval Ávila. Trabajo infantil e inasistencia escolar. Revista Brasileira de Educação 34. Enero-abril. Págs. 68-80. en el abandono a los procesos de formación, ubicando a los países en la escala del subdesarrollo. ${ }^{13}$

Por la naturaleza propia de ser humano, el individuo necesita espacios para su formación y esparcimiento. En lo que a los infanto-jóvenes se refiere, no obstante ser sujetos de derechos, las familias y los Estados tienen el deber de brindarles las condiciones necesarias para un desarrollo integral. ${ }^{14}$ Lamentablemente, se observa que algunos menores consideran que el trabajo cotidiano es un estado normal, e incluso, en aquellos casos en que realizan ventas callejeras, consideran que estas no representan trabajo, llegando a laborar desde edades tempranas con la anuencia de los padres. ${ }^{15}$ Pero, de modo general, es posible identificar formas de trabajo infantil que constituyen manifestaciones alienantes y dañinas para los niños que se ven involucrados en este tipo de actividades, tales como la delincuencia, la sobreexplotación y la prostitución infantil. ${ }^{16}$ Sólo cuando los niños se acercan a la adolescencia, empiezan a ser conscientes de su discriminación por ser menores trabajadores, cuando sus compañeros de estudio los llaman en forma displicente "placeros". ${ }^{17}$

La posibilidad de devengar dinero por trabajar también representa un riesgo, ya que puede fomentar la deserción escolar: el joven empieza a sentir la necesidad del dinero y la responsabilidad de conseguirlo. Socialmente, estos infanto-jóvenes pasan de ser protegidos a proteger a su familia económicamente. Otro de los riesgos asociados al trabajo infantil es que a futuro, cuando esta población se haga adulta, las oportunidades de empleo se restringen a trabajos poco calificados y de bajos salarios, contribuyendo de esta manera a reproducir los esquemas de organización familiar y las condiciones de pobreza. ${ }^{18}$

En esencia, el trabajo infantil causa consecuencias negativas en el niño, y en sus procesos y desarrollo físico, psíquico, educativo y social. También le impide gozar plenamente con sus pares del derecho a la educación, el juego y la salud. Esto se convierte en un círculo vicioso que lo único que logra es reproducir la pobreza.

13. Pedraza, supra, nota 9.

14. Véase Unicef. Despertando conciencia junto con la soCIEDAD CIVIL PARA LA ERRADICACIÓN Y PREVENCIÓN DEL TRABAJO Infantil. Ministerio de Trabajo, Empleo y Seguridad Social, Ministerio de Educación, Ciencia y Tecnología y Unicef. (2005).

15. Véase Ruth Hernández Páez, Ana Cecilia Hernández y Yuriam Lida Rubiano Mesa. Representaciones sociales del trabajo infantil. Orinoquia 1. 2005. Págs. 19- 29.

16. Véase Serrano, Mesa y Mármol, supra, nota 8.

17. Véase Hernández, Hernández y Rubiano, supra, nota 15.

18. Véase Sandoval, supra, nota 12 


\section{El trabajo del menor en la economía familiar}

La situación de pobreza justifica en la sociedad la actividad laboral de los niños articulando diferentes sectores económicos. ${ }^{19}$ De hecho, la situación socioeconómica de las familias impide la permanencia en el sistema educativo de los menores o dificulta su rendimiento escolar. ${ }^{20}$ Algunos modelos educativos plantean que la decisión de trabajar por parte de los menores le compete a los padres, quienes son los que deciden si pueden dedicarse a estudiar, trabajar o a combinar ambas actividades, enfatizando que la familia tiene un papel importante. ${ }^{21} \mathrm{Y}$, aunque muchas veces la situación económica de la familia no se va a ver mejorada porque el menor trabaje, sí puede darse una mejoría en las relaciones intrafamiliares ya que se suavizan si hay algún alivio económico. Considerando esta perspectiva, el joven trabajador asistirá a la escuela y no trabajará cuando la utilidad marginal de estudiar exceda la utilidad marginal de trabajar en cualquiera de las categorías. ${ }^{22}$

En realidad, se debe analizar cada caso en concreto para saber si verdaderamente esta relación costo-beneficio del trabajo infantil favorece al núcleo familiar. ${ }^{23} \mathrm{Al}$ respecto, existen diferentes hipótesis teóricas y modelos que intentan explicar la repercusión del medio en el menor y su relación con la maximización de su utilidad, así como la utilidad del hogar, de manera que resulta necesario evaluar si los retornos por educación que perciben los jóvenes versus los salarios percibidos en el mercado son verdaderamente productivos. ${ }^{24}$ Lamentablemente, han sido identificadas formas de trabajo infantil que son alienantes y dañinas para los menores, y que pueden desencadenar prácticas de delincuencia y que sean víctimas de sobreexplotación. ${ }^{25}$ Incluso, desde las familias por la autoridad imperante. ${ }^{26}$

19. Véase Fernando Maureira Estrada. Trabajo infantil. Algunas consideraciones desde la antropología. Revista Austral De CienCias Sociales 6. 2002. Págs. 113-123.

20. Véase Sandoval, supra, nota 12.

21. Cf. Isabel Chaparro Camargo. Relaciones de parentalidad y conyugalidad presentes en un grupo de familias en situación de trabajo infantil. Desarrollo, Economía y Sociedad 1. Enero-diciembre de 2002. Págs. 27-34.

22. Véase Sebastián Urueña, Luis Tovar y Maribel Castillo. Determinantes del trabajo infantil y la escolaridad: el caso del Valle del Cauca en Colombia. Revista Latinoamericana de Ciencias Sociales, NiÑEZ y Juventud 2. 2009. Págs. 707-733.

23. Véase Pedraza y Ribero, supra, nota 1.

24. Véase Urueña, Tovar y Castillo, supra, nota 22.

25. Véase Maureira, supra, nota 19.

26. Véase Vanessa Romero Mendoza et al. Factores familiares y socia-

les de alto riesgo asociados al trabajo infantil en ciudades de la Costa
El trabajo infantil y juvenil no disminuye notoriamente los índices de pobreza de las familias. ${ }^{27}$ Particularmente en Colombia, desde el 2008 debido a distintos problemas que ha atravesado el país, numerosos niños y jóvenes han tenido que unirse a la vida laboral. ${ }^{28}$ Sin embargo, para el caso de familias de escasos recursos, el desempleo de uno de los padres o de la cabeza económica de la familia propicia la incorporación de los niños a actividades productivas, incluyendo los escenarios presentados anteriormente y llevando en ocasiones a la mendicidad y a la venta callejera, entre otras modalidades. ${ }^{29}$

\section{Metodología DEL ESTUdio}

El nombre de la institución educativa y del municipio donde se realizó la investigación se mantiene en reserva, en atención a los principios de confidencialidad del estudio. Sin embargo, es importante destacar que la población rural involucrada participó activamente en la formulación y validación de las estrategias más adecuadas para el cumplimiento de los procesos formativos de los estudiantes. La muestra de estudio estuvo conformada por estudiantes de noveno a once, pertenecientes a la cadena productiva familiar, a quienes se les hizo seguimiento mediante un diario de campo y entrevistas personalizadas. En el proceso de recolección de información, intervinieron las autoridades académicas de la institución educativa, y en la transferencia de las estrategias participaron los estudiantes, la comunidad, e incluso la Policía Nacional.

\section{IDENTIFICACIÓN DE ESTRATEGIAS}

\section{PARA APOYAR EL PROCESO DE FORMACIÓN DEL MENOR}

Por parte de la normatividad colombiana, persiste el interés de diseñar estrategias para prevenir y erradicar el trabajo infantil. ${ }^{30} \mathrm{~A}$ nivel local, hay evidencias de lo que se hace desde colegios y entes de Gobierno

Caribe colombiana. Universitas Psychologica 2. Abril-junio de 2012. Págs. 481-496.

27. Véase Cepal. Repercusiones de la Educación y del TrabaJO DE Niños y Adolescentes. (1995). Disponible en: http://www. cepal.org/publicaciones/xml/9/.../PS1995_CapituloII-v4.pdf

28. Véase Romero et al. supra, nota 26.

29. Véase Maureira, supra, nota 19.

30. Véase Ministerio de Educación Nacional. Plan SECTORIAL 2010-2014. Documento N.o 9. (2011). 
para promocionar el "No al trabajo infantil", tales como campañas y promoción por periódicos y radio. La mayoría de las acciones realizadas en el ámbito rural pasan desapercibidas. ${ }^{31}$ Se hace necesario plantear también estrategias que permitan mitigar las consecuencias negativas causadas por este trabajo durante el proceso de formación de los jóvenes.

\section{A. Ajustes al currículo}

En varias regiones del mundo, se encuentra una falta de pertinencia de los programas de formación con el entorno y, por ende, se señalan deficiencias en la cualificación de los docentes responsables del proceso. Los esquemas educativos, en su mayoría, están descontextualizados y alejados de la realidad campesina, es decir, el currículo está diseñado para el sector urbano, por lo cual se requieren ajustes al currículo. ${ }^{32}$ En esencia, se necesita un análisis profundo, cambios oportunos e interés gubernamental para que los procesos educativos y las entidades que los brindan mejoren favorablemente. ${ }^{33}$

La idea inicial de realizar cambios al currículo requiere de una alta complejidad y reflexión por cuanto se deben cumplir las normas que rigen las estructuras curriculares. Sin embargo, es posible hacer ajustes a las estrategias pedagógicas que se implementen en el aula si se tienen en cuenta las utopías que se pretenden alcanzar y las verdaderas prácticas que vive el maestro con el estudiante en el interior del aula de clase. ${ }^{34}$ Se pueden introducir cambios pedagógicos que respondan significativamente a los procesos de enseñanza-aprendizaje, lo cual exige un trabajo mayor por parte del maestro, quien debe mantener una actitud de reflexión permanente sobre su quehacer, de modo que oriente sus prácticas hacia un trabajo en el que intervenga el colectivo del aula y que sus relaciones intrapersonales aporten a las transformaciones que se requieren con urgencia. En esencia, tener presente lo que dice Gómez, ${ }^{35}$ el maes-

31. Andrew Pearse. Problemas estructurales de sistemas educativos en América Latina. Revista Latinoamericana de Estados EduCATivos 3-4. Tercer-cuarto trimestres del 2006. Págs. 319-350.

32. Id.

33. Véase María de Ibarrola Nicolín. Los grandes problemas del sistema educativo mexicano. Perfiles educativos Número Especial 2012. Págs. 16-28.

34. Véase Serrano, Mesa y Mármol, supra, nota 8.

35. Cf. Alberto Gómez. Tradiciones curriculares, innovaciones educativas y función social conservadora del conocimiento escolar: primacía de los temas sobre los problemas. Revista DE TeORÍA Y Didáctica sobre las Ciencias Sociales 7. Enero-diciembre de 2002. Págs. 221-246. tro debe interiorizar que hay vida dentro de la escuela y que el apoyo del grupo puede aportar al proceso de aprendizaje.

Por otro lado, contrario a lo señalado también por Gómez ${ }^{36}$ de acuerdo con la experiencia de campo evidenciada en la comunidad rural donde se trabajó, no es cierta la generalización según la cual es común la falta de disposición de los profesores formados en una disciplina para abordar los temas en profundidad; lo que hace falta son procesos de cualificación docente hacia nuevas técnicas de enseñanza acordes con las necesidades del entorno. Aquí se hace fundamental que el docente conozca el contexto, la cultura, las costumbres y aspectos generales de una comunidad antes de llevar a cabo su trabajo pedagógico, ya que los estudiantes necesitan sentirse identificados y cómodos en el ambiente escolar, donde el respeto por las diferencias se evidencie en cada una de las relaciones interpersonales, involucrando en ello a maestro y educandos. ${ }^{37}$ En términos generales, a fin de cumplir estos fines, las estrategias pedagógicas se convierten en el pilar dentro del quehacer docente para transformar el contexto escolar en un verdadero taller de aprendizaje.

\section{B. Creación de programas abiertos y a distancia}

Nicolín ${ }^{38}$ generaliza que los programas abiertos y a distancia son poco eficaces, ya que se han acrecentado y no han arrojado cambios significativos. Sin embargo, se deben reconocer los avances y el apoyo educativo que ha brindado este tipo de oferta de formación en los cursos ofrecidos por el Servicio Nacional de Aprendizaje (senA), entidad que de manera virtual y a distancia apoya, cualifica y certifica a los educandos. Este tipo de apoyo educativo de estrategia de enseñanza puede implementarse con el fin de mejorar las condiciones de formación en las comunidades rurales y, aun así, sirve para las urbanas. Se requiere nuevamente el compromiso de las instituciones gubernamentales, ya que esto implicaría una mejora en la conectividad de las zonas rurales, y en la formación a los docentes y capacitación a los estudiantes, de manera que mediante grupos de apoyo, chats y tareas dirigidas pudieran alcanzarse algunos logros no obtenidos por las ausencias de los estudiantes. ${ }^{39}$ Sólo con un Estado comprometido se pueden lograr cambios que rompan con el paradigma

\footnotetext{
36. Id.

37. Véase Serrano, Mesa y Mármol, supra, nota 8.

38. Véase Nicolín, supra, nota 33.

39. Véase Serrano, Mesa y Mármol, supra, nota 8.
} 
según el cual en América Latina poco se invierte en salud, seguridad social y educación. Una reformulación del gasto público educativo llevaría por ende a nuevas políticas educativas que respondan a las verdaderas necesidades de los jóvenes y del país. ${ }^{40}$

Este tipo de orientación no sustituye la presencialidad, pero sí aporta herramientas para subsanar las deficiencias que se puedan generar por el ausentismo. La educación a distancia requiere de un aprendizaje autónomo que implica un alto compromiso y responsabilidad individual de quien se educa. Se necesita entonces adquirir una cultura frente a la responsabilidad de cada sujeto en su proceso de formación; sin ese elemento no existiría transformación, el estudiante tiene que participar de ello en cuestión de fondo y forma.

\section{Talleres de sensibilización}

La familia representa un sistema de múltiples variables y niveles de interacción entre sus miembros. Algunos factores del ambiente familiar, la estructura de las familias, las condiciones socioeconómicas, el nivel de escolaridad de los padres y las expectativas de los padres para con sus hijos deben considerarse al momento de plantear estrategias encaminadas a disminuir el trabajo infantil. ${ }^{41}$ Es así como a la familia se le considera uno de los agentes mediadores para que los niños y las niñas se relacionen con la sociedad adquiriendo valores, normas y conductas que les servirán para el desarrollo de sus operaciones mentales, lo que lleva también a que parte de la conducta de un niño se estructure desde allí. ${ }^{42}$

Por lo tanto, se hace necesario desarrollar talleres de sensibilización con la comunidad adulta, con el fin de concientizar a los mayores sobre los riesgos de trabajos infantiles, y particularmente de aquellos trabajos reiterativos y prolongados.

La OIT diseñó un taller de sensibilización para padres de familia en el que se aborda el tema del trabajo infantil, así como cuáles son los derechos de niños y niñas que pueden verse afectados con esta práctica, desmejorando su calidad de vida. Este taller comprende tres momentos: 1) la exploración de conocimientos de los padres de familia sobre este tópico y la socialización de experiencias personales; 2) la orientación y reflexión del facilitador, en busca de que los padres de familia contrasten lo que saben con lo que se les está diciendo, y 3) llevar a la práctica lo orientado a través de estrategias como la elaboración de carteleras, cartas, firma de compromisos, etc.

En la comunidad rural en donde se realizó el estudio, se llevó a cabo el taller de sensibilizacion siguiendo las recomendaciones de la огт. Este taller culminó con una campaña contra el trabajo infantil en la zona, gracias a la cual los docentes tuvieron en cuenta el diagnóstico de necesidades de la región y de experiencias laborales de los jóvenes. Los estudiantes que participaron presentaron sus vivencias y las de otros compañeros y, apoyados de carteles y arengas, se recorrió el centro del pueblo. El hecho de que los estudiantes se vincularan evidenció el impacto que pueden producir los talleres de esta naturaleza en los mismos adolescentes víctimas del trabajo infantil y juvenil. Igualmente, hace suponer que en padres de familia también puede haber un impacto significativo desde las escuelas de padres si se trabajan talleres que lleven a la crítica reflexiva sobre las consecuencias de esta práctica. ${ }^{43}$

Los estudiantes, desde su iniciativa y originalidad, explicaron lo que para ellos significa el trabajo infantil (véase gráfico 1 , gráfico 2 y gráfico 3), mediante un desfile realizado por las calles principales del pueblo, generando conciencia en los habitantes. Las opiniones de los estudiantes reflejan que no comparten que los niños trabajen porque consideran que ganan menos trabajando que estudiando (gráfico 1). Sin embargo, a pesar de que para ellos no hay ganancia, la representación del niño trabajador no demuestra desagrado hacia lo que está haciendo.
40. Véase Alejandro Márquez Jiménez. El financiamiento de la educación en México. Problemas y alternativas. Perfiles Educativos. 2012. Págs. 107-117.

41. Cf. Sherin Shearin. Kinship care placement and children's academic performance. Journal of Health \& Social Policy 3-4. 2007. Pages. 31-43.

42. Véase Gladys Villarroel y Ximena Sánchez. Relación familia y escuela: un estudio comparativo en la ruralidad. Estudios PedagóGICOS 28. 2002. Págs. 123-141.
43. Véase Ministerio de Educación Nacional. Guía N. ${ }^{\circ}$ 26: ¿Cómo PARTICIPAR EN LOS PROCESOS EDUCATIVOS DE LA ESCUELA? Corpoeducación. (2007). 


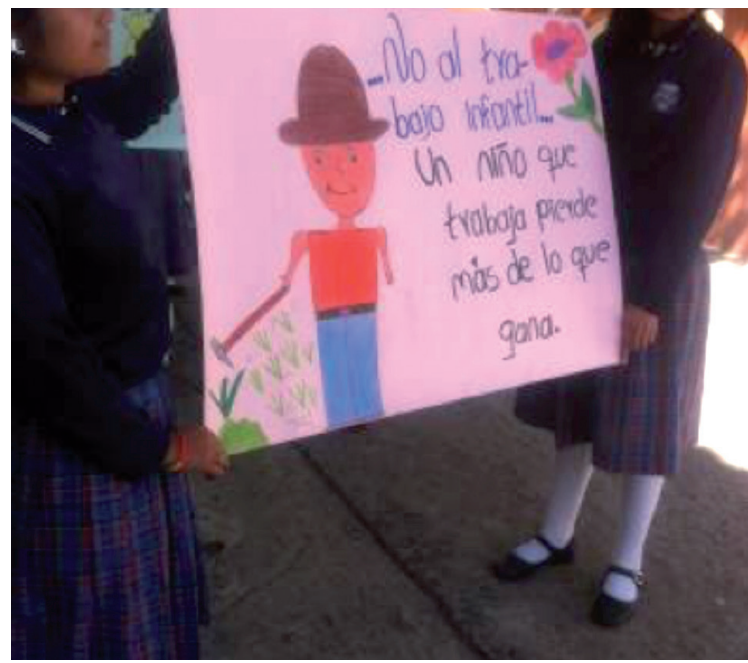

Gráfico 1. Expresión gráfica con alusión escrita de la postura de los estudiantes hacia el trabajo infantil

Fuente: elaboración propia

Otros mensajes incluidos en los afiches preparados para el desfile reflejaron la necesidad de la educación antes que el trabajo, resaltando la importancia de este derecho para la vida de los menores (gráfico 2), así como otros manifestaron la consigna universal según la cual los niños son el futuro y, por lo tanto, el trabajo infantil debe ser erradicado (gráfico 3).

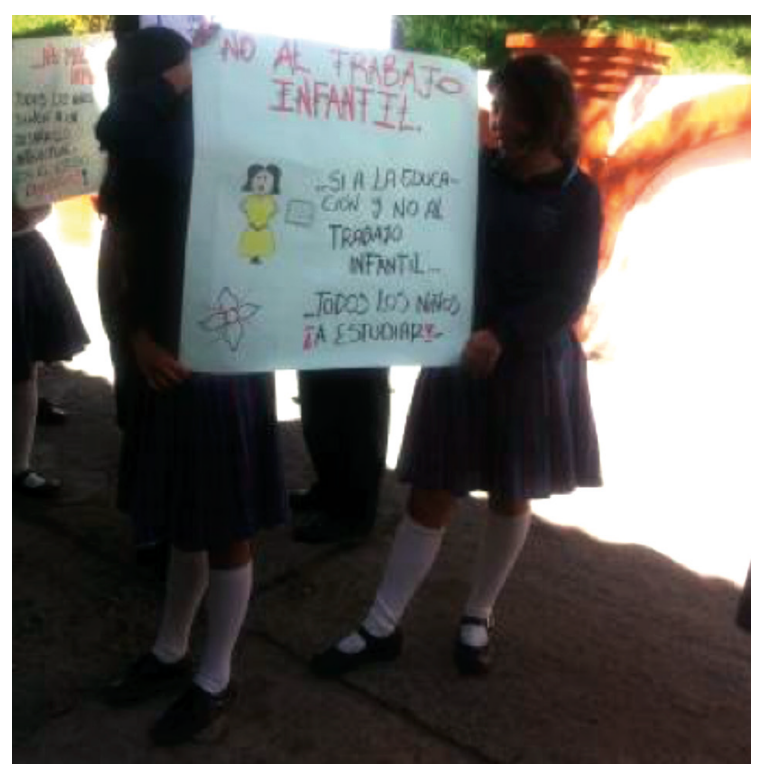

Gráfico 2. Invitación de los niños a estudiar antes que trabajar Fuente: elaboración propia

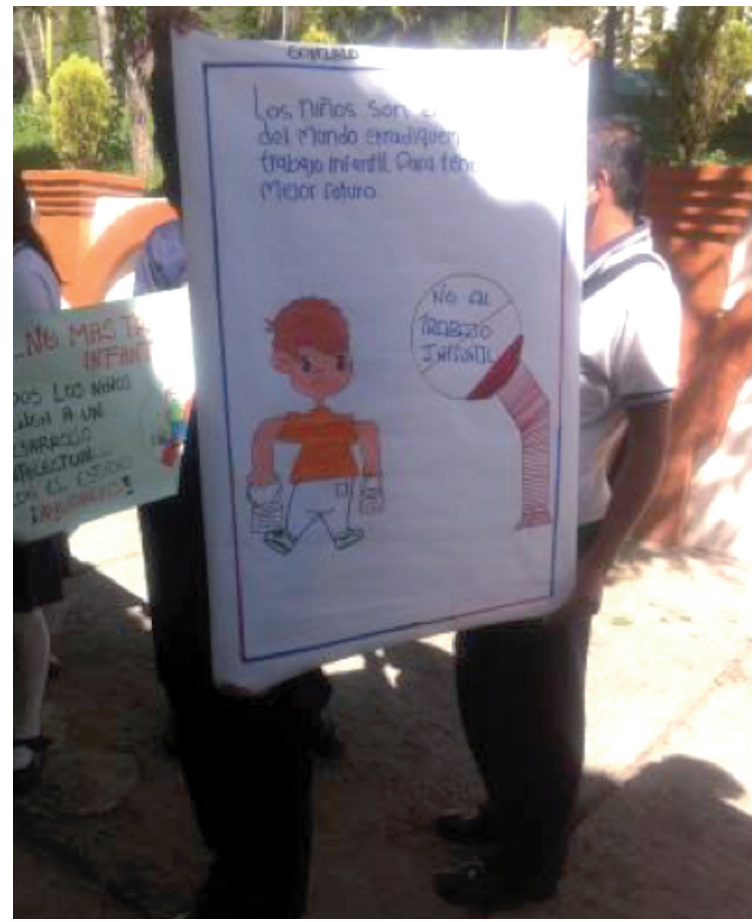

Gráfico 3. Representación de enfado e inconformidad de un niño obligado al trabajo infantil

Fuente: elaboración propia

En el ámbito regional, y a nivel de Colombia, las comisarías de familia y las instancias encargadas de velar por los derechos de niños, niñas y adolescentes pueden también organizar y llevar a cabo los talleres de sensibilización, dando cumplimiento así a la Estrategia Nacional para Prevenir y Erradicar el Trabajo Infantil y Proteger al Joven Trabajador. ${ }^{44}$ Esta estrategia inicia con la recolección de datos que den fe de la situación de vulneración de los derechos de estos niños y adolescentes, a través de un trabajo mancomunado con la institución educativa. Con esta base de datos, la comisaría plantea las medidas urgentes teniendo en cuenta el grado de riesgo en que se pueda encontrar el menor de edad trabajador. Posteriormente, se dará el acompañamiento permanente de esta instancia a niños, niñas y adolescentes y a sus padres, mediante capacitaciones, talleres, asesorías, etc. La Procuraduría General de la Nación debe conocer sobre estas medidas tomadas.

44. Véase Ministerio del Trabajo y unAD. Metodología PARA LA implementaCión de La Estrategia NaCiOnal PARA PREVENIR Y Erradicar el Trabajo Infantil y Proteger al Joven TrabaJADOR 2008-2015. Ministerio del Trabajo (2011). 


\section{Aprendizaje basado en problemas y trabajo colaborativo}

Diariamente, los estudiantes se enfrentan a retos académicos y sociales propios de su entorno, por lo cual el rendimiento escolar puede afectarse por estas interacciones. ${ }^{45}$ Han sido desarrollados numerosos modelos que tratan de explicar estas interacciones sin encontrar uno que sea universal, justamente porque la condición social del ser humano varía de una región a otra y entre edades.

Se debe tener en cuenta a todos los integrantes del sistema sin ignorar el impacto que se logra con las acciones de algunos y las negaciones a actuar de otros. La motivación que hace que el grupo funcione está dada por sus propios componentes. En un trabajo colaborativo es necesario que se agencien los procesos de manera participativa y solidaria, previa definición de un interés común y la convicción de que es necesario su cumplimiento. ${ }^{46}$ Un ambiente propicio para desarrollar un trabajo colaborativo implica:

- Disponer momentos para escuchar y entender al otro.

- Conjugar las diferencias y reconocer cualidades de otros.

- Generar espacios para la reflexión, coordinación y evaluación de acciones.

- Proponer un proyecto común.

- Procurar el diálogo y los acuerdos para tomar decisiones conjuntas.

- Proponer responsabilidades a los participantes para que ellos escojan.

- Crear vínculos afectivos para compartir experiencias propias.

- Generar espacios para aprender de los demás.

El aprendizaje basado en problemas es un proceso de aprendizaje que gira en torno al planteamiento de una situación problemática previamente diseñada o una real, y la elaboración de constructos.

45. Gregory Liem y Andrew Martin. Peer relationships and adolescents' academic and non-academic outcomes: same-sex and opposite-sex peer effects and the mediating role of school engagement. British Journal of Educational Psychology 81. 2011. Pages 183-206.

46. Véase Maritza Díaz, Gabriela Luna y Mónica Tobón. MateriaLES DE TRABAJO PARA PREVENIR Y ERRADICAR EL TRABAJO INFANTIL DOMÉstico. Organización Internacional del Trabajo. (2004).
Este modelo de aprendizaje ofrece beneficios tales como: ${ }^{47}$

- Los alumnos desarrollan habilidades y competencias tales como colaboración, planeación de proyectos, comunicación, toma de decisiones y manejo del tiempo.

- Aumentan la motivación. Se registra un aumento en la asistencia a la escuela, mayor participación en clase y mejor disposición para realizar las tareas.

- Integración entre el aprendizaje en la escuela y la realidad. Los estudiantes retienen mayor cantidad de conocimiento y habilidades cuando están comprometidos con proyectos estimulantes. Se hace énfasis en cuándo y dónde se pueden utilizar en el mundo real.

- Desarrollo de habilidades de colaboración para construir conocimiento. El aprendizaje colaborativo permite a los estudiantes compartir ideas entre ellos, expresar sus propias opiniones y negociar soluciones, habilidades todas necesarias en los futuros puestos de trabajo.

- Acrecentar las habilidades para la solución de problemas.

- Establecer relaciones de integración entre diferentes disciplinas.

- Aumentar la autoestima.

- Acrecentar las fortalezas individuales de aprendizaje y de sus diferentes enfoques y estilos hacia este.

\section{Conclusiones}

La educación no es una responsabilidad que recae en el grupo de maestros que ejercen su quehacer; esta es un compromiso de todos que inicia en la voluntad política de los Gobiernos para mejorar la calidad y las condiciones tanto físicas y de infraestructura, como de mejoramiento del recurso humano para la cualificación docente.

El trabajo infantil causa consecuencias negativas en el niño, y en sus procesos y desarrollo físico, psíquico, educativo y social. También le impide gozar plenamente con sus pares del derecho a la educación, el juego y la salud. Esto se convierte en un círculo vicioso que lo único que logra es reproducir la pobreza. Los niños tienen derecho a una calidad de vida digna durante la infancia, entendiendo esta

47. Véase Lourdes Galeana. Aprendizaje basado en proyectos. InVEStigación en Educación a Distancia, Revista Digital. (2006). Disponible en: http://ceupromed.ucol.mx/revista/ 
como la época que transcurre entre el nacimiento y la edad adulta. Sin embargo, factores económicos obligan a los niños a formar parte de la cadena productiva familiar, y cuando esta implica trabajo fuera de casa, se corre el riesgo de menores salarios y condiciones precarias para laborar, a lo cual se añaden los bajos niveles de escolaridad de los padres, quienes a pesar de ello han subsistido y no ven la formación de sus hijos como una prioridad. A fin de que el niño viva plenamente sus derechos, su núcleo familiar debe de vivir en un ambiente de dignidad humana. De lo contrario, se cumpliría con maquillar la realidad social.

Por ser multicausal el trabajo infantil, es importante tener en cuenta que no sólo factores económicos llevan a los menores de edad a trabajar. El papel de la familia en la vida de estos niños y jóvenes es crucial para que ellos deban o no trabajar. Las características de una familia es un punto a tenerse en cuenta en el aumento o disminución de los índices de trabajo infantil.

Las estrategias planteadas pueden llegar a generar un gran impacto y cambio social, siempre y cuando se logre también un cambio en la cultura del trabajo infantil en el pueblo. En relación con los ajustes al currículo, resultaría interesante que el Ministerio de Educación tuviese en cuenta que Colombia maneja diversos contextos y cómo, en ocasiones, lo impuesto por el Gobierno no puede ser camisa de fuerza para quienes no tienen las herramientas y facilidades de acceso al conocimiento. Los docentes podrían ajustar sus planes de aula y de clase a las necesidades del medio como algunos lo han venido haciendo, pero el problema se daría cuando alguno de los estudiantes acceda a la educación superior y encuentre barreras para la aplicación de lo aprendido.

De acuerdo con lo manifestado, la educación abierta y a distancia es una posible solución, ya que sus ofertas académicas en su mayoría analizan las necesidades del medio. En relación con los talleres de sensibilización, sería aconsejable vincular a toda la comunidad, de manera que siendo los mismos estudiantes los multiplicadores de las experiencias, le presenten a todas las instancias del municipio su percepción frente al trabajo infantil. Es importante que los padres de familia se incluyan en el cambio de cultura porque es desde la familia que los hijos adquieren actitudes y conductas. Finalmente, con la pedagogía del aprendizaje basado en problemas y el trabajo colaborativo, los estudiantes que trabajan desarrollarían sus operaciones mentales y se adaptarían al currículo.

\section{REFERENCIAS}

Alberto Gómez. Tradiciones curriculares, innovaciones educativas y función social conservadora del conocimiento escolar: primacía de los temas sobre los problemas. Revista de Teoría y Didáctica sobre las Ciencias Sociales 7. Enero-diciembre de 2002. Págs. 221-246.

Alejandro Márquez Jiménez. El financiamiento de la educación en México. Problemas y alternativas. Perfiles Educativos. 2012. Págs. 107-117.

Andrew Pearse. Problemas estructurales de sistemas educativos en América Latina. Revista LatinoamericANA DE Estados Educativos 3-4. Tercer-cuarto trimestre de 2006. Págs. 319-350

Antonio Sandoval Ávila. Trabajo infantil e inasistencia escolar. Revista Brasileira de Educação 34. Enero-abril. Págs. 68-80.

Aura Pedraza y Rocío Ribero. El trabajo infantil y juvenil en Colombia y algunas de sus consecuencias claves. REvisTA LATINOAmericana de Ciencias Sociales, NiÑEZ y Juventud 1. Enero-junio de 2006. Págs. 2-28.

Cepal. RePERCusiones DE LA EDUCACión Y DEL TRABAJO DE NIÑOS Y ADOLESCENTES. (1995). Disponible en: www.cepal.org/publicaciones/xml/9/.../PS1995_CapituloII-v4.pdf

Constitución Política de Colombia. Artículo 44. Julio 7 de 1991. (Colombia).

Departamento Nacional de Planeación. Hacia la prosperidad democrática: visión 2010-2014. BASES DEL PLAN Nacional de Desarrollo 2010-2014. Departamento Nacional de Planeación. (2011).

Fernando Maureira Estrada. Trabajo infantil. Algunas consideraciones desde la antropología. Revista Austral DE Ciencias Sociales 6. 2002, 113-123.

Gladys Villarroel y Ximena Sánchez. Relación familia y escuela: un estudio comparativo en la ruralidad. EstuDios Pedagógicos 28. 2002. Págs. 123-141.

Isabel Chaparro Camargo. Relaciones de parentalidad y conyugalidad presentes en un grupo de familias en situación de trabajo infantil. Desarrollo, Economía y SocieDAD 1. Enero-diciembre de 2002. Págs. 27-34.

Gregory Liem y Andrew Martin. Peer relationships and adolescents' academic and non-academic outcomes: same-sex and opposite-sex peer effects and the mediating role of school engagement. BRITISH JOURNAL OF EDUCational Psychology 81. 2011. Pages 183-206.

Lourdes Galeana. Aprendizaje basado en proyectos. InvESTIGACión en Educación a Distancia, Revista Digital. (2006). Disponible en: http://ceupromed.ucol.mx/revista/ 
María de Ibarrola Nicolín. Los grandes problemas del sistema educativo mexicano. Perfiles educativos Número EsPecial 2012. Págs. 16-28.

Maritza Díaz, Gabriela Luna y Mónica Tobón. Materiales DE TRABAJO PARA PREVENIR Y ERRADICAR EL TRABAJO INFANTIL DOMÉSTICO. Organización Internacional del Trabajo. (2004).

Ministerio de Educación Nacional. Guía No. 26: ¿Cómo PARTICIPAR EN LOS PROCESOS EDUCATIVOS DE LA ESCUELA? Corpoeducación. (2007).

Ministerio de Educación Nacional. Plan SECTORIAL 20102014. Documento N. ${ }^{\circ}$ 9. (2011).

Ministerio del Trabajo y unAD. Metodología PARA LA IMPLEMENTACión DE LA Estrategia NaCional PARA Prevenir y Erradicar el Trabajo Infantil y ProTeger AL Joven Trabajador 2008-2015. Ministerio del Trabajo. (2011).

Mónica Serrano, Marisol Mesa y Marina del Mar Mármol. IDENTIFICACIÓN DE INTERACCIONES ACADÉMICAS Y FAMILIARES QUE EVIDENCIAN CAMBIOS EN EL DESEMPEÑO ACADÉMICO DE ADOLESCENTES QUE INTERVIENEN EN LA CADENA PRODUCTIVA FAMILIAR EN UNA COMUnidad RURal. Tesis de Maestría. Fundación Universitaria Católica de Colombia. (2014).

Organización Internacional del Trabajo. Fortalecimiento A FAMILIAS CON NIÑOS, NIÑAS Y JÓVENES TRABAJADORES. UN CAMINO PARA LA PREVENCIÓN Y ERRADICACiÓN DEL TRABAJO INFANTIL EN COLOMBia. (2005). Disponible en: http://www.ilo.org/ipec/facts/lang--es/ index.htm

Presidencia de la República. Presidente Santos Resalta informe del Departamento de Trabajo de EEUU
SOBRE AVANCES DE COLOMBIA PARA ELIMINAR LAS PEORES FORMAS DE TRABAJO INFANTIL. (Octubre 1 de 2013). Disponible en: http://wsp.presidencia.gov.co/ Prensa/2013/Octubre/Paginas/2013100104-Presidente-Santos-informe-Departamento-Trabajo-EEUUColo mbia-eliminar-peores-formas-trabajo-infantil.aspx

Ruth Hernández Páez, Ana Cecilia Hernández, Yuriam Lida Rubiano Mesa. Representaciones sociales del trabajo infantil. Orinoquia 1. 2005. Págs. 19- 29.

Sebastián Urueña, Luis Tovar y Maribel Castillo. Determinantes del trabajo infantil y la escolaridad: el caso del Valle del Cauca en Colombia. Revista LATINOAMERICana de Ciencias Sociales, Niñez y Juventud 2. 2009. Págs. 707-733.

Sherin Shearin. Kinship care placement and children's academic performance. Journal of Health \& Social PoliCy 3-4. 2007. Pages 31-43.

Silvio Feldman, Emilio García y Hege Araldsen. Los NiÑos QUE TRABAJAN. Unicef Argentina. (1997).

Unicef. Despertando CONCIENCIA JUNTO CON LA SOCIEDAD CIVIL PARA LA ERRADICACIÓN Y PREVENCIÓN DEL trabajo infantil. Ministerio de Trabajo, Empleo y Seguridad Social, Ministerio de Educación, Ciencia y Tecnología y Unicef. (2005).

Vanessa Romero Mendoza et al. Factores familiares y sociales de alto riesgo asociados al trabajo infantil en ciudades de la Costa Caribe colombiana. Universitas PsychoLOGICA 2. Abril-junio de 2012. Págs. 481-496.

Zandra Pedraza Gómez. El trabajo infantil en clave colonial: consideraciones histórico-antropológicas. REVISTA NóMADAS 26. Abril de 2007. Págs. 80-90. 